\title{
Simultaneous VEP and PERG investigations in early Parkinson's disease
}

\author{
S Calzetti, A Franchi, G Taratufolo, E Groppi
}

\begin{abstract}
To evaluate whether visual evoked potential (VEP) and pattern electroretinogram (PERG) abnormalities may be detectable early in the course of Parkinson's disease (PD) and to assess a possible interdependence between retinal and cortical potentials, simultaneous VEP and PERG were carried out in nine patients with early PD. The mean (SD) duration of the disease was 10 (6) months. None of the patients had been previously treated with levodopa or other antiparkinsonian drugs. The results show VEP and PERG abnormalities that are spatial stimulus dependent, with higher frequencies being more involved and also indicate that VEP changes are not entirely dependent on alterations at the retinal level.
\end{abstract}

Parkinson's disease (PD) is characterised by a widespread deficiency of the neurotransmitter dopamine (DA) in the central nervous system. ${ }^{1}$ A deficiency of DA is also likely to involve the multisynaptic visual pathway resulting in abnormal visual evoked potential (VEP). ${ }^{2}$

Recently, pattern electroretinogram (PERG) abnormalities have been reported in PD suggesting a primary retinal dysfunction partially responsible for the changes in the VEPs. ${ }^{3-5}$ However, the PERG changes were mainly in patients in the advanced stages of PD with dissimilar previous drug treatments. The initial site of dopaminergic dysfunction in the visual system which could be detected early in the course of the disease is unclear.

The aim of this study is to investigate whether simultaneous recordings of both VEP and PERG abnormalities may be found in the early stages of PD.

Istituto di Neurologia and Istituto di Oftalmologia Università di Parma, Italy

A Franchi

A Franchi

E Groppi

Correspondence to: Dr Stefano Calzetti, Istitutó di Neurologia Via del Ouartiere 4 , 43100 Parma, Italy.

Received 17 February 1989 and in revised form 27 July 1989

Accepted 16 August 1989 Nine patients with early PD, four male and five female, mean age 64 (SD) 12.5 years, were studied. The mean duration of the disease was 10 (SD) 6 months. The mean lag time between the diagnosis of the disease and the electrophysiological investigations was 1.9 (SD) 1.6 months. The severity of the disease was rated according to Hohen and Yahr stages (seven patients were classified as having stage II and two patients as having stage III). The right side was more affected in five patients and the left side in the remaining. None had been previously treated with levodopa or other antiparkinsonian drugs.
Twelve age-matched healthy subjects, seven male and five female, were taken as controls. All patients and controls were free from ophthalmological diseases and had a corrected visual acuity equal or better than 0.8 in each eye.

\section{Stimuli}

Steady-state and transient VEP and PERG responses were examined. For steady-state response a checkerboard stimulus was counterphase modulated at a rate of 4 and $8 \mathrm{~Hz}$, producing 8 and 16 pattern reversals per second, respectively. For transient response the stimulus was counterphase modulated at a rate of $2.7 \mathrm{~Hz}(5.4$ pattern reversals per second).

Three spatial frequencies were employed $2.44,1.74,0.87$ cycles/degree (c/d). The contrast of the checkerboard (defined as the difference between maximum and minimum luminance divided by the sum) was $97^{\circ}{ }_{0}$. The stimulus field was circular and subtended a visual angle of $9^{\circ}$ at the eye on examination. The mean screen luminance was $60 \mathrm{~cd} / \mathrm{m}^{2}$. The luminance of the surround was matched to the luminance of the screen.

\section{PERG and VEP measurement}

VEPs were recorded using the active $\mathrm{AgCl}$ electrode placed at the $\mathrm{Oz}$ and the reference electrode placed at the $\mathrm{Cz}$. The PERGs were recorded placing the active $\mathrm{AgCl}$ electrodes on the skin over the lower eyelid. The reference electrode was taped over the eyelid of the contralateral unstimulated eye to reduce the noise coming from conjugate eye movements and muscular activity from the eyelid. The central forehead was grounded.

Signals were amplified (100000 fold for PERG and 20000 fold for VEP), filtered between 1 and $30 \mathrm{~Hz}$ and averaged 200 times by a signal processor, allowing rejection of single sweeps disturbed by artifacts. The analysis time was $250 \mathrm{~ms}$.

According to previous studies ${ }^{67}$ steady-state 
Figure 1 An example of simultaneous PERG and $V E P$ recordings obtained in a $P D$ patient. Stimulus parameters: $2.44 c / d, 5.4$ rev/sec. $A=$ Amplitude; $L=$ Latency. $P$, indicates the first positive wave, $N$, indicates the first negative wave.

Figure 2 (A) An example of steady-state (4 Hz at $1.74 \mathrm{cld})$ PERG and VEP obtained in a $P D$ patient. (B) Fast Fourier transform (FFT) of the response; the amplitude of each frequency component is proportional to the height of the vertical bars. (C) Digitally filtered $2^{\text {nd }}$ harmonic $(8 \mathrm{~Hz})$ component of the pattern response.
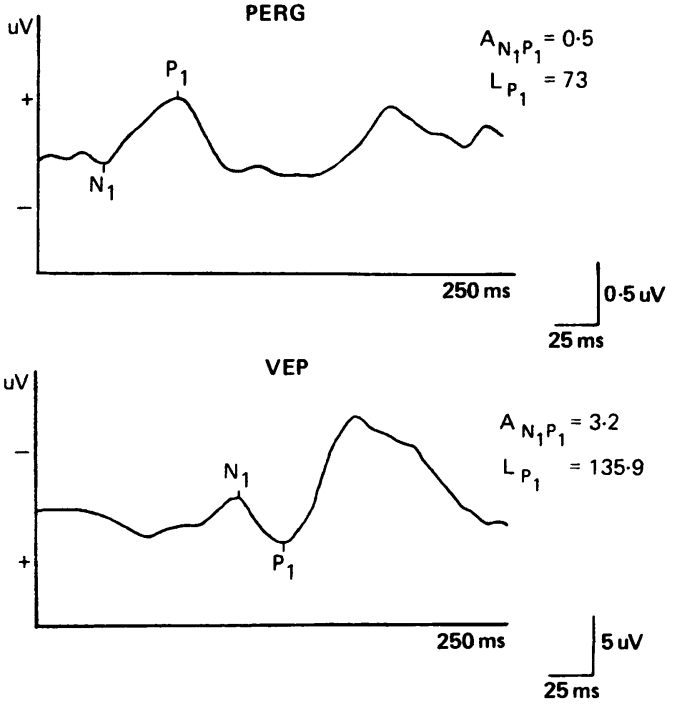

second harmonic of the response was measured (fig 2).

Procedure

The examination was performed monocularly and with the natural pupil and the subjects were asked to fixate on a small red spot placed in the centre of the monitor at a distance of 100 $\mathrm{cm}$. Simultaneous PERG and VEP to 1,4 and 8 $\mathrm{Hz}$ stimulation rate was measured in a single session. A second session was necessary to record PERG and VEP in the contralateral eye. The three stimulation rates were randomly presented. Between each recording a three minute interval was allowed to avoid adaptation effects.
Statistical analysis (two tailed significance level) was performed by using the Student $t$ test for comparison with the control group, Wilcoxon's rank test for comparison of the intraindividual variables and Spearman's rank correlation.

\section{Results}

Visual Evoked Potential (VEP)

There was a significant difference in VEP amplitude between PD patients and controls for both transient and steady-state responses only for one of the spatial frequencies tested, that is, $2 \cdot 44 \mathrm{c} / \mathrm{d}$ (table 1 ).

VEP latency was found to be significantly different between PD patients and controls at two spatial frequencies tested, that is, 1.74 and $2.44 \mathrm{c} / \mathrm{d}$ (table 1 ). Table 2 shows the number of eyes whose VEP amplitude and latency differed by more than 2 SD from the mean control values for each spatial and temporal frequency examined.

Pattern Electroretinogram (PERG)

Mean PERG amplitude did not differ significantly between PD patients and controls for both transient and steady-state temporal frequencies at the three spatial frequencies tested (table 1). However, in some patients PERG amplitude was found reduced as compared to mean control values for both transient and steady-state stimulation. There was a significant difference in the latency of transient PERG between PD and controls only for one spatial frequency, that is, $2.44 \mathrm{c} / \mathrm{d}$ (table 1 ).

Table 2 summarises the number of eyes

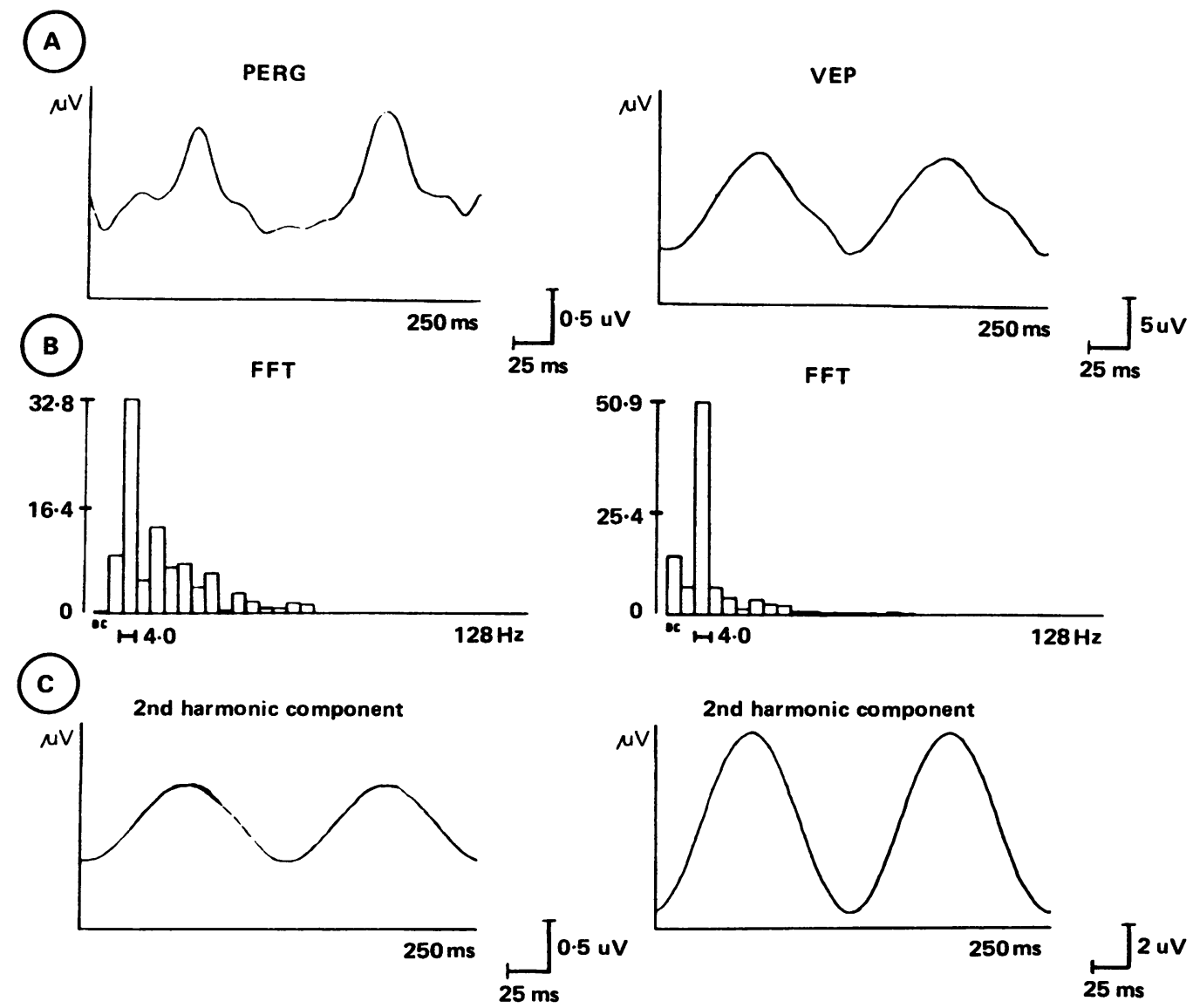


Table 1 Mean values (SD) of VEP and PERG amplitude (MV) and latency (ms) in PD patients and controls

\begin{tabular}{|c|c|c|c|c|c|}
\hline & \multicolumn{5}{|c|}{ TEMPORAL FREQUENCY } \\
\hline & \multicolumn{2}{|l|}{ VEP Amplitude } & & \multicolumn{2}{|l|}{ PERG Amplitude } \\
\hline & PD patients & controls & & $P D$ patients & controls \\
\hline $\begin{array}{l}2 \cdot 44 \\
1 \cdot 74 \\
0.87 \\
2 \cdot 44 \\
1.74 \\
0.87 \\
2 \cdot 44 \\
1 \cdot 74 \\
0.87\end{array}$ & $\begin{array}{l}1.95(1.07)^{\star} \\
4 \cdot 5(3 \cdot 21) \\
2 \cdot 8(2 \cdot 62) \\
4 \cdot 71(2 \cdot 91)^{\star} \\
9 \cdot 7(4 \cdot 13) \\
9 \cdot 35(3 \cdot 56) \\
5 \cdot 04(2 \cdot 49)^{\ddagger} \\
6.3(4 \cdot 7) \\
5.9(2 \cdot 84)\end{array}$ & $\begin{array}{l}4 \cdot 04(1) \\
5 \cdot 35(2 \cdot 05) \\
4 \cdot 13(1 \cdot 67) \\
8(1 \cdot 75) \\
10 \cdot 6(1 \cdot 84) \\
10(1 \cdot 88) \\
5 \cdot 6(2 \cdot 1) \\
6 \cdot 9(3 \cdot 1) \\
6 \cdot 4(2 \cdot 47)\end{array}$ & $\begin{array}{l}(16 \mathrm{rev} / \mathrm{sec}) \\
(8 \mathrm{rev} / \mathrm{sec}) \\
(5.4 \mathrm{rev} / \mathrm{sec})\end{array}$ & $\begin{array}{l}0.4(0.24) \\
0.61(0.22) \\
0.74(0.29) \\
0.45(0.3) \\
0.67(0.33) \\
0.77(0.33) \\
0.82(0.26) \\
1.12(0.57) \\
1.17(0.6)\end{array}$ & $\begin{array}{l}0.52(0.17) \\
0.64(0.23) \\
0.74(0.27) \\
0.6(0.18) \\
0.8(0.22) \\
0.9(0.13) \\
1.02(0.20) \\
1.18(0.21) \\
1.25(0.28)\end{array}$ \\
\hline $\begin{array}{l}2 \cdot 44 \\
1 \cdot 74 \\
0 \cdot 87\end{array}$ & $\begin{array}{l}\text { VEP Latency } \\
132 \cdot 5(11 \cdot 5)^{\dagger} \\
121 \cdot 2(6 \cdot 25)^{\star} \\
109 \cdot 1(6 \cdot 4)\end{array}$ & $\begin{array}{l}123.5(2.57) \\
113.4(3.3) \\
109 \cdot 2(5.4)\end{array}$ & $(5.4 \mathrm{rev} / \mathrm{sec})$ & $\begin{array}{l}\text { PERG Latency } \\
70(5 \cdot 2)^{\star} \\
65(3 \cdot 2) \\
66(2 \cdot 89)\end{array}$ & $\begin{array}{l}64(2 \cdot 19) \\
63 \cdot 3(2 \cdot 1) \\
63 \cdot 4(1 \cdot 88)\end{array}$ \\
\hline
\end{tabular}

${ }^{\star} \mathrm{p}<\cdot 01,+\mathrm{p}<\cdot 02 \pm \mathrm{p}<\cdot 05$ as compared to controls.

whose PERG amplitude and latency was found abnormal (by more than $2 \mathrm{SD}$ ) as compared to mean control values for each spatial and temporal frequency examined.

In $P D$ patients no significant correlation was found between VEP and PERG latency at the only stimulus configuration $(2.44 \mathrm{c} / \mathrm{d}, 5.4 \mathrm{rev} /$ sec) found abnormal in both tests. The age of the PD patients was not correlated with either amplitude or latency of both VEP and PERG, except for VEP at $5.4 \mathrm{rev} / \mathrm{sec}$ with $1.74 \mathrm{c} / \mathrm{d}$ $(\mathrm{rs}=76, \mathrm{p}<0.01$, number of eyes $=17$ ).

When the amplitude and latency of VEP and PERG of the more and the less affected side were compared in each PD patient, lower values of VEP amplitude were found in the more affected side only for $8 \mathrm{rev} / \mathrm{sec}$ temporal frequency at $2.44 \mathrm{c} / \mathrm{d}(\mathrm{p}<0.05, \mathrm{n}=8)$ and a more delayed PERG latency was found in the more affected side at $2.44 \mathrm{c} / \mathrm{d}(\mathrm{p}<0.05, \mathrm{n}=8)$.

\section{Discussion}

Electrophysiological changes in the visual system have been reported in Parkinson's disease.$^{2-58}$ However, these abnormalities have been found mainly in patients with fully developed disease, most of whom had been previously exposed to levodopa and/or other dopaminergic drugs. Therefore, it remains unclear whether or not VEP and PERG abnormalities may occur in the early stages of the disease.

To our knowledge this is the first report of simultaneous "steady-state" and "transient" VEP and PERG recordings at different spatial frequencies obtained in patients with early

Table 2 Number of eyes whose VEP and PERG amplitude and latency is abnormal in PD patients (Total number of eyes examined $=17$ )

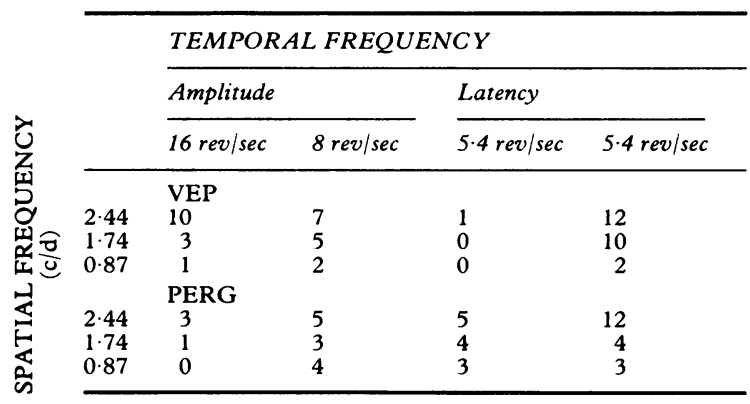

Parkinson's disease and previously untreated with levodopa and other antiparkinsonian drugs.

The results of this study indicate that VEP and PERG abnormalities may be detected in early stages of PD depending on the stimulus configuration. Spatial selectivity of the stimulus has also been demonstrated recently in PD patients which failed to show abnormal VEP with spatial frequency stimuli below $1 \mathrm{c} / \mathrm{d}^{9}{ }^{10}$ Our findings on VEP amplitude and latency abnormalities at spatial frequency above $1.7 \mathrm{c} / \mathrm{d}$ are consistent with the previous reports.

It has been suggested that there is a greater sensitivity of grating as compared to checkerboard pattern in VEP examination of PD patients. ${ }^{91112}$ Our study indicates that significant VEP abnormalities may also be observed with checkerboard stimulus that agrees with other studies. ${ }^{513}$ It remains unclear whether the retina is the site primarily involved in the dysfunction of the visual pathway in PD. Our findings indicate a different degree of involvement of VEP and PERG responses resulting from the same temporal and spatial stimulus, VEP being more affected than PERG. This supports the hypothesis of an earlier dysfunction of the central visual pathway, that may be relatively independent of the abnormalities at the retinal level.

As far as we know, only three studies on PERG in PD patients have been reported. ${ }^{3-5}$ Our data on PERG amplitude agree with the report of Gottlob et $a l,{ }^{5}$ in which PERG amplitude obtained with comparable spatial and temporal frequency ( $25 \mathrm{~min}$ of arc, $2 \mathrm{rev} /$ sec) was found to be normal at high contrast level $\left(97^{\circ}\right)$. This contrasts with the results of Nightingale $e t a l,{ }^{3}$ who found a normal PERG amplitude by using a 25 minute check size and reduced PERG values with a 50 minute check size at $95^{\circ}{ }_{0}$ contrast. This is difficult to equate with recent experimental studies on the effect of DA depletion on single cell activities of the retina. ${ }^{1415}$ These studies have provided evidence that selective spatial properties of the stimulus determines the detection of visual changes in retinal DA pathway, with only higher spatial frequencies being affected.

Moreover, recently Ghilardi et $a l^{16}$ showed abnormalities in both PERG amplitude and, to 
a lesser degree, latency in MPTP-induced Parkinsonism in monkeys only for 1.2 or above c/d stimulus configuration.

A delay in PERG latency restricted to higher spatial frequency $(2 \mathrm{c} / \mathrm{d})$ has been previously reported by Stanzione $e t a l^{4}$ in 19 PD patients, whose clinical features were not fully detailed. The failure in detecting PERG latency abnormalities reported by Nightingale et $a l^{3}$ is possibly due to a lower spatial stimulus configuration and agrees with our findings. In contrast, an earlier PERG latency, at $97^{\circ}$ o contrast, as reported by Gottlob et al is not easily explained.

The finding of an interocular difference in VEP amplitude and PERG latency restricted to the highest spatial frequencies examined with the more affected side showing more marked electrophysiological abnormalities, suggests a possible correlation between motor and visual pathways impairment that may be already detectable in the early stages of Parkinson's disease. This agrees with reports by BodisWollner et al. ${ }^{17}$

In summary the main findings of this study are: VEP changes are not entirely dependent on alterations in the retina; PERG latency is a suitable index for detecting retinal changes early in the course of PD; VEP and PERG abnormalities are spatial stimulus dependent, with higher frequencies being more involved in the early stages of PD.

1 Barbeau A, Campanella G, Butterworth R, Yamada $\mathrm{K}$. Uptake and efflux of $14 \mathrm{C}$-dopamine in platelets. Evidence for a generalised defect in Parkinson's disease. Neurology for a general

2 Bodis-Wollner I, Yahr MD. Measurements of visual evoked potentials in Parkinson's disease. Brain 1978;101:661-71. Nightingale S, Mitchell KW, Howe JW. Visual evoked cortical potential and pattern electroretinograms in Parkinson's disease and control subjects. J Neurol Neurosurg Psychiatry 1986;49:1280-7.

4 Stanzione P, Pierelli F, Stefano E, Peppe A, Rizzo PA Morocutti C. Simultaneous recording of pattern electroretinogram and visual evoked potentials in patients with Parkinson's disease. EEG and Clin. Neurophysiol 1987;S 100 (abstract).

5 Gottlob I, Schneider E, Heider W, Skrandies W. Alteration of visual evoked potentials and electroretinograms in Parkinson's disease. EEG and Clin. Neurophysiol 1987; 66:349-57.

6 Regan D. Steady-state evoked potentials. J Opt Soc Am 1977;67:1475-89.

7 Regan D. Comparison of transient and steady-state methods. Ann NY Acad Sci 1982;388:45-71.

8 Kupersmith MJ, Shakin E, Siegel IM, Lieberman A. Visual system abnormalities in patients with Parkinson's disease. Arch Neurol 1982;39:284-6.

9 Tartaglione A, Pizio N, Bino G, Spadavecchia L, Favale E. VEP changes in Parkinson's disease are stimulus dependent. J Neurol, Neurosurg Psychiatry 1984;47:305-7.

10 Onofri M, Ghilardi MF, Basciani M, Gambi D. Visual evoked potentials in Parkinsonism and dopamine blockade reveal a stimulus-dependent dopamine function in humans. J Neurol Neurosurg Psychiatry 1986;49:1150-9.

11 Bhaskar PA, Vanchilingam S, Amala Bhaskar E, Devaprabhu A, Ganesan RA. Effect of l-dopa on visual evoked potential in patients with Parkinson's disease. Neurol 1986;36:1119-21.

12 Bodis-Wollner I, Onofri MC, Marx MS, Mylin LH. Visual Evoked Potentials in Parkinson's Disease: spatial frequency, temporal rate, contrast and the effect of frequency, temporal rate, contrast and the effect of dopaminergic drug. In: Alan R,

13 Dinner DS, Luders H, Hanson M, Lesser RP, Klem G. Pattern evoked potentials (PEP's) in Parkinson's disease. Neurology 1985;35:610-3.

14 Cohen JL, Dowling JE. The role of retinal interplexiform cell: Effect of 6-hydroxydopamine on the spatial properties of carp horizontal cell. Brain Res 1983;264:307-10.

15 Teranishi T, Negishi K, Kato S. Regulatory effect of dopamine on spatial properties of horizontal cells in carp retina. $J$ Neurosci $1984 ; 4: 1271-80$

16 Ghilardi MF, Bodis-Wollner I, Onofri MC, Marx MJ, Glover AA. Spatial frequency-dependent abnormalities of the pattern electroretinogram and visual evoked potentials

17 Bodis-Wollner I, Yahr MD, Thornton J. Visual Evoked Potentials and the severity of Parkinson's disease. In: Clifford RF and Calpideo R, eds. Progress in Parkinson's Disease. New York: Pitman Medical, 1981:126-37. 